\title{
The Asymptotic Chebyshev Coefficients for \\ Functions with Logarithmic Endpoint Singularities: \\ Mappings and Singular Basis Functions
}

John P. Boyd

Department for Atmospheric \& Oceanic Science

and Laboratory for Scientific Computation

University of Michigan

2455 Hayward Avenue

Ann Arbor, Michigan 48109

Transmitted by Melvin R. Scott

\section{ABSTRACT}

When a function is singular at the ends of its expansion interval, its Chebyshev coefficients $a_{n}$ converge very poorly. We analyze three numerical strategies for coping with such singularities of the form $(1 \pm x)^{k} \log (1 \pm x)$, and in the process make some modest additions to the theory of Chebyshev expansions. The first two numerical methods are the convergence-improving changes of coordinate $x=\sin [(\pi / 2) y]$ and $x=\tanh \left[L y /\left(1-y^{2}\right)^{1 / 2}\right]$. We derive the asymptotic Chebyshev coefficients in the limit $n \rightarrow \infty$ for both mappings and for the original, untransformed Chebyshev series. For the original function, the asymptotic approximation for general $k$ is augmented by the exact Chebyshev coefficients for integer $k$. Numerical tests show that the sine mapping is excellent for $k \geqslant 1$, increasing the rate of convergence to $b_{n}=O\left(1 / n^{4 k+1}\right)$. Although the tanh transformation is guaranteed to be better for sufficiently large $n$, we offer both theoretical and numerical evidence to explain why the sine mapping is usually better in practice: "sufficiently large $n$ " is usually huge. Instead of mapping, one may use a third strategy: supplementing the Chebyshev polynomials with singular basis functions. Simple experiments show that this approach is also successful.

\section{INTRODUCTION}

Solutions to differential equations often are singalar at corners and endpoints. Several examples are discussed by Lund and Riley [13] and Lee, Schultz, and Boyd [12]. Unfortunately, these boundary branch points seri- 
ously degrade the efficiency of Chebyshev-polynomial methods. In this article, we limit ourselves to the particular case of logarithmic endpoint singula.ities of the form

$$
(1 \pm x)^{k} \log (1 \pm x)
$$

and pursue a twofold goal. The first is to quantify this poor convergence by proving asymptotic approximations to the Chebyshev coefficients. The second is to analyze three numerical strategies for improving the efficiency of Chebyshev expansions for such singular functions.

The primary tool for the asymptotic analysis is the method of steepest descent $[3,4]$, which is applicable for general $k$, where $k$ is the exponent of the power of $x$ multiplying the logarithm. However, we also derive the exact coefficients for (1.1) for integer $k$ in Section 2. Similar asymptotic estimates for functions with fractional-power branch points at $x= \pm 1$ have been previously obtained by Elliott [9].

The first two numerical strategies are to apply a mapping of the form

$$
x=f(y)
$$

and then use a Chebyshev series in the new coordinate $y$. The two particular transformations we analyze are (1) $x=\sin [(\pi / 2) y]$, which is representative of mappings that vary algebraically with $y$ near the endpoints (Section 3 ), and (2) $\left.x=\tanh \left[L y /\left(1-y^{2}\right)^{! / 2}\right)\right]$, which is discussed in Section 4 and is representative of mappings that vary exponentially fast near $x= \pm 1$. The steepest-descent method again yields asymptotic approximations for the coefficients.

In Section 5, we apply the asymptotic formulas to reduce the error in cailculating Chebyshev coefficients via Gauss-Chebyshev quadrature. Section 6 uses a mixture of the asymptotic estimates with numerical experiments to explain why the tanh mapping, which would seem to be the best option in view of its superior performance in the limit $n \rightarrow \infty$, is usually inferior to the sine mapping (or no change of coordinate at all) unless $n$ is very large indeed.

The third numerical strategy is an alternative to mapping: using a mixed basis set composed of Chebyshev polynomials plus a few singular basis functions (Section 7). The heuristic justification for this strategy is that if the coefficients of the singular functions match those of the branch points in $f(x)$, then the $T_{n}(x)$ are only required to approximate the nonsingular portion of $f(x)$.

The final section is a summary and a comparison of the three numerical methods for coping with endpoint singularities. 


\section{LOGARITHMIC ENDPOINT SINGULARITIES WITHOUT A MAPPING}

THEOREM 1. If a function $f(x)$ has a singularity at $x=1$ of the form

$$
\begin{aligned}
f(x)= & \alpha(1-x)^{k} \log (1-x)+\beta(1+x)^{k} \log (1+x) \\
& + \text { less singular terms, }
\end{aligned}
$$

where $k$ is positive and $\alpha$ and $\beta$ are arbitrary constants, then the coefficients of the Chebyshev series

$$
f(x)=\sum_{n=0}^{\infty} a_{n} T_{n}(x)
$$

are asymptotically given by

$$
a_{n} \sim-\left[\alpha+\beta(-1)^{n}\right] 2^{1-k} \Gamma(2 k+1) n^{-(2 k+1)} d_{n}(k)[1+O(1 / n)]
$$

with

$$
d_{n} \equiv \cos k \pi+\frac{2}{\pi}(\sin k \pi)[\psi(2 k+1)-\log n]
$$

where $\psi$ is the usual digamma function. For the special case of integer $k$, $d_{n}=(-1)^{k}$ and the relative error is $O\left(1 / n^{2}\right)$ instead of $O(1 / n)$.

Proof. The usual integrals for the Chebyshev coefficients

$$
a_{n} \equiv \frac{2}{\pi} \int_{-1}^{1} f(x) T_{n}(x)\left(1-x^{2}\right)^{-1 / 2} d x
$$

$(n>0)$ may be transformed by the substitution $x=\cos t$, which converts the $n$th Chebyshev polynomial in $x$ into $\cos n t$, to give

$$
a_{n}=\frac{2}{\pi} \int_{0}^{\pi} f(\cos t) \cos n t d t
$$


Since the Chebyshev coefiicients are asymptotically dominated by the worst singularities, that is, the branch points whose lowest derivatives are unbounded, it follows that the coefficient integrals must be dominated by the neighborhood of the endpoints. Consequently, we may legitimately approximate (2.5) by integrals that are simpler to evaluate so long as the endpoint singularities are unaltered. For simplicity, set $\beta=0$, so that the $f(\cos t)$ is singular only at $x=1$, that is, $t=0$.

By elementary Taylor expansion,

$$
f(\cos t) \approx 2^{1-k} t^{2 k} \log t+\text { less singular terms. }
$$

Since this is accurate only for small $t$, one may replace the upper limit of integration by anything which is convenient so long as the integrand is nonsingular. Thus, asymptotically

$$
a_{n}(k) \sim \frac{2^{2-k}}{\pi} \int_{0}^{1} t^{2 k} \log t \cos n t d t, \quad n \gg 1
$$

The case $k=-0$ has been used to illustrate the method of steepest descents in the textbook by Bender and Orszag [1], but their method is applicable for general $k$. The key idea is to make the replacement $\cos n t=\operatorname{Re} e^{i n t}$ and then deform the contour of integration into three line segments: (i) $t=0$ to $t=i \infty$, (ii) $t=i \infty$ to $t=1+i \infty$, and (iii) $t=1+i \infty$ to $t=1$. The integral around the segment at infinity is zero. The integral along the third segment may be evaluated by Watson's lemma to show that it is $O\left(1 / n^{2 k+2}\right)$, which is a factor of $n$ smaller than the integral along (i). Thus, the dominant contribution comes from the integral

$$
a_{n}(k)-\frac{2^{2-k}}{\pi} \operatorname{Re}\left(i^{2 k+1} \int_{0}^{\infty}(\log i s) e^{-n s} s^{2 k} d s\right)
$$

Applying the identity $\log i s=\log s+i \pi / 2$ then converts the integral in ( $\mathbf{Z} . \overline{8})$ into a pair. The first is the integral which defines the gamma function. The second is proportional to

$$
\int_{0}^{\infty}(\log s) e^{-n s} s^{2 k} d s=n^{-(2 k+1)} \Gamma(2 k+1)[\psi(2 k+1)-\log n]
$$

where the RHS is given by identity 4.352 of Gradshteyn and Rhyzik [11]. Adding (2.9) to the gamma-function integral then proves the theorem. 
When $k$ is an integer, the $\log n$ term disappears and we can calculate the exact Chebyshev coefficients through a different line of reasoning. The exact $a_{n}$ for the special case $k=0$ are given (as Fouritr coefficients) by identity 1.441.2 of [11]. By means of two $t$-differentiations of $f(\cos [t])$ and the application of trigonometric identities, the coefficients of $(1-x) \log (1-x)$ may be obtained from those for $\log (1-x)$. Repeating the procedure gives the coefficients of $(1-x)^{k} \log (1-x)$ for arbitrary nonnegative integer $k$. To obtain the corresponding expansions for $(1+x)^{k} \log (1+x)$, merely replace $x$ by $-x$ and exploit the parity of the Chebyshev polynomials: $T_{2 n}(x)=$ $T_{2 n}(-x)$ while $T_{2 n+1}(x)=-T_{2 n+1}(-x)$. One finds, for arbitrary constants $\alpha$ and $\beta$,

$$
\begin{aligned}
\alpha \log (1-x)+\beta \log (1+x) & \\
= & -(\alpha+\beta) \log 2+\sum_{n=1}^{\infty} \frac{-2}{n}\left[\alpha+\beta(-1)^{n}\right] T_{n}(x), \\
& \alpha(1-x) \log (1-x)+\beta(1+x) \log (1+x) \\
= & (\alpha+\beta)(1-\log 2)+(\alpha-\beta)\left(\log 2-\frac{3}{2}\right) T_{1}(x) \\
& +\sum_{n=2}^{\infty}\left[\alpha+\beta(-1)^{n}\right] \frac{2}{n\left(n^{2}-1\right)} T_{n}(x) \\
& \alpha(1-x)^{2} \log (1-x)+\beta(1+x)^{2} \log (1+x) \\
- & (\alpha+\beta)\left(\frac{7}{4}-\frac{3}{2} \log 2\right)+(\alpha-\beta)\left(2 \log 2-\frac{8}{3}\right) T_{1}(x) \\
+ & (\alpha+\beta)\left(\frac{25}{24}-\frac{1}{2} \log 2\right) T_{2}(x) \\
& +\sum_{n=3}^{\infty}\left[\alpha+\beta(-1)^{n}\right] \frac{-12}{n\left(n^{2}-1\right)\left(n^{2}-4\right)} T_{n}(x),
\end{aligned}
$$

Comparing these results with Theorem 1 , we see that the asymptotic approximation is exact for $k=0$ while the relative errors are $O\left(1 / n^{2}\right)$ for $k=1$ and $O\left(5 / n^{2}\right)$ for $f(x)=\alpha(1-x)^{2} \log (1-x)+\beta(1+x)^{2} \log (1+x)$. Thus, the asymptotic expressions are extremely accurate approximations to the exact Chebyshev coefficients even for $n$ as small as 10. (However, the relative error is only $O(1 / n)$ when $k$ is not an integer.) 
Observe that each of (2.10) through (2.12) consists of a finite number of special formulas plus a general term for $n>k$. An anonymous reviewer pointed out that by converting the coefficient integrals to

$$
a_{n}=2\left[\alpha+\beta(-1)^{n}\right](-1)^{k+1} \int_{0}^{\infty} e^{-n s}(\cosh s-1)^{k} d s \quad(n>k)
$$

"using the methods of Elliott [9]," one obtains an integral which may be evaluated via a Laplace transform table:

$a_{n}=\frac{(-1)^{k+1}\left[\alpha+\beta(-1)^{n}\right] 2^{1-k}(n-k-1) !(2 k) !}{(n+k) !} \quad(n>k ; \quad k$ integral $)$

Presumably this could also be deduced via the recursive procedure that gives (2.10) to (2.12).

Unfortunately, this alternative line of proof merely gives the general term in the Chebyshev series; the representation (2.13) is not valid for $n \leqslant k$. The approach through $t$-differentiation and trigonometric identities is still the orly algorithm for computing all the exact coefficients for integer $k$. When $k$ is not an integer, the steepest-descent proof, which yields only an asymptotic approximation, is best one can do. Fortunately, the asymptotic formulas will be quite adequate for the analysis and applications in the rest of the paper.

\section{LOGARITHMIC SINGULARITIES WITH A QUADRATIC MAPPING}

THEOREM 2. If the function

$$
f(x) \equiv \alpha(1-x)^{k} \log (1-x)+\beta(1+x)^{k} \log (1+x)
$$

is expanded in Chebyshev polynomials after the application of the map

$$
x=\sin \frac{\pi}{2} y,
$$

then the coefficients in the series

$$
f(x)=\sum_{n=0}^{\infty} b_{n} T_{n}(y[x])
$$


are asymptotically given by

$$
b_{n} \sim-\frac{\left[\alpha+\beta(-1)^{n}\right] \pi^{2 k} 2^{2-5 k}(4 k) !}{n^{4 k+1}}\left[1+O\left(\frac{1}{n^{2}}\right)\right], \quad n \gg 1 .
$$

Proof. Defining the trigonometric variable $t$ via $y=\cos t$ and Taylor expanding,

$$
\begin{aligned}
x(t) & \equiv \sin \frac{\pi}{2} y \equiv \sin \left(\frac{\pi}{2} \cos t\right) \\
& =1-\frac{\pi^{2}}{32} t^{4}+O\left(t^{6}\right), \quad t \rightarrow 0 .
\end{aligned}
$$

One may then apply the rest of the proof of Theorem 1 .

From (3.6) one can show that the mapping has a "quadratic" character: $1-x \approx\left(\pi^{2} / 8\right)(1-y)^{2}$ near $x=1$, and similarly near the other endpoint. The result is that the asymptotic rate of decrease of the Chebyshev coefficients is increased from $O\left(1 / n^{3}\right)$ to $O\left(1 / n^{5}\right)$ for a function like $(1-x) \log (1-x)$ and from $O\left(1 / n^{5}\right)$ to $O\left(1 / n^{9}\right)$ for $(1-x)^{2} \log (1-x)$. Intriguingly, however, there is no improvement in rate (and a modest increase in the propcrtionality constant) for the "naked" logarithm, $\log (1-x)$.

Table 1 shows that Chebyshev coefficients of the model functions with s'ne mapping tend rapidly to asymptotic limits given in Theorem 2 . No results are given for $\log (1-y)$, because the error in the asymptotic approximation is indistinguishable from zero for $n>6$, indicating that either the approximation is in fact exact (except for the lowest three or four coefficients), or has a relative error which is $O\left(1 / n^{4}\right)$.

\section{HYPERBOLIC-TANGENT MAPPING}

The rationale for the tanh map, which was first suggested by F. Stenger [14], is that if the interval $x \in[-1,1]$ is mapped to ihe unbounded interval $z \in[-\infty, \infty]$ through a transformation that uses the exponential function, weak endpoint singularities are neutralized. Here, "weak" means that $f(x)$ is bounded at $x= \pm 1$. "Neutralized" means that the endpoint zeros are transformed into exponentially fast decay of $f(x[z])$ as $|z| \rightarrow \infty$. If we apply an expansion in $z$, one can prove that the series will converge exponentially 
TABLE 1

EXACT AND APPROXIMATE CHEBYSHEV COEFFICIENTS ${ }^{\mathrm{a}}$ FOR THE MODEL FUNCTIONS $f(y)=(1-y)^{2} \log (1-y)$ AND $f(y)=(1-y) \log (1-y)$ AFTER APPLICATION OF THE CHANGE OF COORDINATE $y=\sin [(\pi / 2) x]$

\begin{tabular}{|c|c|c|c|c|}
\hline \multirow[b]{2}{*}{$n$} & \multicolumn{2}{|c|}{$a_{n}$} & \multirow[b]{2}{*}{ Rel. error } & \multirow[b]{2}{*}{ Rel. error $\times n^{2}$} \\
\hline & Exact & Asymptotic & & \\
\hline \multicolumn{5}{|c|}{ (a) $f(x)=\{1-\sin [(\pi / 2) x]\}^{2} \log \{1-\sin [(\pi / 2) x]\}$} \\
\hline 5 & 0.036258 & $-7.85507 \mathrm{E}-3$ & 1.21664 & 30.4161 \\
\hline 10 & $6.44692 \times-5$ & $-1.53419 \mathrm{~s}-5$ & 1.23797 & 123.797 \\
\hline 15 & $-4.44693 \mathrm{E}-7$ & $-3.99079 \mathrm{E}-7$ & 0.102575 & 23.0794 \\
\hline 20 & $-3.20506 \mathrm{E}-8$ & $-2.99647 \mathrm{E}-8$ & $6.50808 \mathrm{E}-2$ & 26.0323 \\
\hline 25 & $-4.20699 \mathrm{E}-9$ & $-4.0218 \mathrm{E}-9$ & 0.044021 & 27.5131 \\
\hline 30 & $-8.04751 \mathrm{E}-10$ & $-7.79451 \mathrm{E}-10$ & $3.14387 \mathrm{E}-2$ & 28.2948 \\
\hline 35 & $-1.99335 E-10$ & $-\mathbf{1} .94656 \mathrm{E}-10$ & $2.34752 \mathrm{E}-2$ & 28.7571 \\
\hline 40 & $-5.96083 \mathrm{E}-11$ & $-5.85248 \mathrm{E}-11$ & $1.81766 \mathrm{E}-2$ & 29.0825 \\
\hline \multicolumn{5}{|c|}{ (b) $f(x)=\{1-\sin [(\pi / 2) x]\} \log \{1-\sin [(\pi / 2) x]\}$} \\
\hline 5 & $-2.79429 \mathrm{E}-2$ & $-9.47482 E-3$ & 0.660922 & 16.523 \\
\hline 10 & $-3.08103 E-4$ & $-2.96088 E-4$ & $3.89953 \mathrm{E}-2$ & 3.89953 \\
\hline 15 & $-3.97982 E-5$ & $-3.8991 \mathrm{E}-5$ & $2.02809 E-2$ & 4.56321 \\
\hline 20 & $-9.36426 \mathrm{~s}-6$ & $-9.25275 E-6$ & $1.19077 \mathrm{E}-2$ & 4.76307 \\
\hline 25 & $-3.05566 \mathrm{E}-6$ & $-3.03194 \varepsilon-6$ & $7.7615 \mathrm{E}-3$ & 4.85094 \\
\hline 30 & $-1.22514 \mathrm{E}-6$ & $-1.21847 \mathrm{E}-6$ & $5.4416 \mathrm{lE}-3$ & 4.89745 \\
\hline 35 & $-5.66018 \mathrm{E}-7$ & $-5.63743 \mathrm{E}-7$ & $4.02047 \mathrm{E}-3$ & 4.92507 \\
\hline 40 & $-2.90045 \mathrm{E}-7$ & $-2.89149 \mathrm{E}-7$ & $3.08927 \mathrm{E}-3$ & 4.94284 \\
\hline 45 & $-1.6085 \mathrm{E}-7$ & $-1.60457 \mathrm{E}-7$ & $2.44687 E-3$ & 4.95491 \\
\hline 50 & $-9.49367 E-8$ & $-9.47482 \mathrm{E}-8$ & $1.98541 \mathrm{E}-3$ & 4.96353 \\
\hline 55 & $-5.8929 \varepsilon-8$ & $-5.88312 \mathrm{E}-8$ & $1.64291 \mathrm{E}-3$ & 4.9698 \\
\hline 60 & $-3.81299 E-8$ & $-3.80772 \varepsilon-8$ & $1.38181 \mathrm{E}-3$ & 4.97451 \\
\hline
\end{tabular}

a The listed coefficients are those of the Chebyshev series in the new coordinate $x$. The expected relative error is $O\left(p / n^{2}\right)$ for some constant $p$; the fourth column gives the relative error multiplied by $n^{2}$ to confirm this theoretical prediction; the entries converge to $\boldsymbol{p}$ as $\boldsymbol{n} \rightarrow \infty$.

fast because $f(x[z])$ is analytic for all real, finite $z$ and decays exponentially. Instead of the coefficients decreasing asymptotically as some inverse power of $n$ (no map), the error falls as an exponential function of the truncation $N$-that is, falls faster than $1 / N^{k}$ for any finite $k$. One may then map the series from $z$ back to $y \in[-1,1]$.

Stenger [14] suggested sinc functions in $z$, but Boyd [2] pointed out that the alternative of "rational Chebyshev" functions $\operatorname{TB}_{n}(z)$ is just as good. 
Boyd's choice is equivalent to the change of coordinate

$$
x=\tanh \left(\frac{L y}{\left(1-y^{2}\right)^{1 / 2}}\right), \quad x, y \in[-1,1]
$$

followed by an expansion in ordinary Chebyshev polynomials in the new coordinate $y$. The constant $L$ is a user-choosable "map parameter." [The $\mathrm{TB}_{n}(z)$ are the images of the Chebyshev polynomials under the map from $z$ to $y$.] The coefficients of the Chebyshev series in $y$ are identical with those of the $\operatorname{TB}_{n}(z)$ expansion, so one may borrow the asymptotic analysis from Boyd $[3,4]$.

Paradoxically, the tanh mapping (4.1), which has the most rapid rate of convergence in the asymptotic limit $N \rightarrow \infty$, is markedly inferior to the sine mapping or even no mapping at all unless (1) the singularity is strong ( $k$ small) or (2) one needs many decimal places of accuracy. In consequence, we shall not recapitulate [3] and [4] in detail, but instead focus on why a change of coordinate which is so good in theory is often poor in practice.

Boyd [3] shows that the asymptotic Chebyshev coefficients of a function $g(z)$ on an unbounded interval are the sum of two types of contributions. First, there are residue terms which are generated by the singularities of $g(z)$ in the finite $z$-plane. Second, there are steepest descent, or endpoint, terms which depend upon the rate at which $g(z)$ decays as $|z| \rightarrow \infty$. The residue contributions decrease as exponentials whose arguments are linear in $N$. This has been dubbed "geometric" convergence, since the terms of an ordinary geometric series have the same property with each term smaller than its predecessor by a factor of $1 / \delta$, where $\delta>1$. In contrast, the endpoint contributions decay as exponentials of a fractional power of $n$-"subgeometric" convergence in the parlance of [3]. Asymptotically, the "resiảu" contributions can be ignored.

Unfortunately, for finite $n$-sometimes even for very large $n$-the "residue" terms may be important, or even dominant. Figure 1 illustrates the coefficients of the Chebyshev series (in $y$ ) for $f(x)=(1-x)^{2} \log (1-x)$ after the mapping (4.1) is applied (solid curve). The asymptotic analysis of Boyd [3] shows that as $n \rightarrow \infty$, these coefficients are (with $L=3$ ) of the form

$$
a_{n} \sim \exp (-0.48 n) r(n)+\exp \left(-3.46 n^{1 / 2}\right) q(n)
$$

where $r(n)$ and $q(n)$ are complicated and oscillatory but $O(1)$ functions which are irrelevant to our main purpose, which is to bound the magnitude of the Chebyshev coefficients. The long dashes in Figure 1 show 


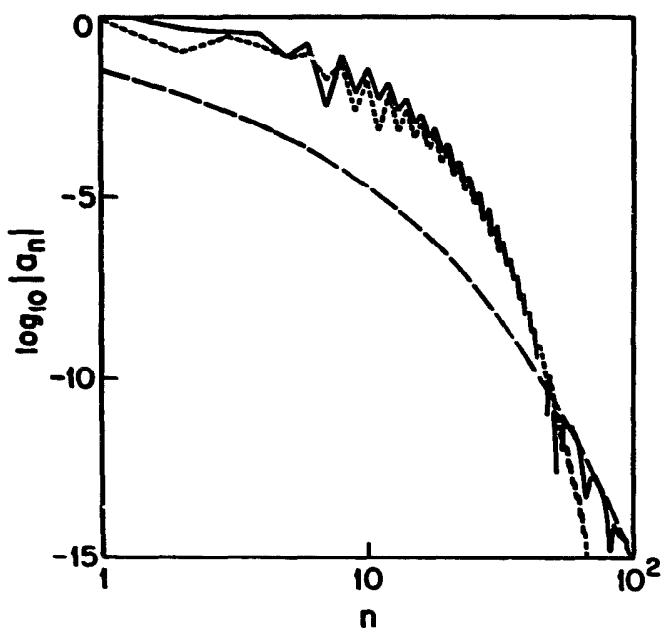

Fic. 1. Solid curve: exact Chebyshev coefficients for $f(x)=(1-x)^{2} \log (1-x)$ after application of the tanh map with $L=3$. Long dashes: "amplitude" of the endpoint contributions to the asymptotic Chebyshev coefficients of this function. Short dashes: exact coefficients of a function $g(y[x])$ obtained by modifying the tanh mapping so as to preserve the poles on the imaginary axis while removing the endpoint singularities.

$\exp \left(-3.46 n^{1 / 2}\right)$. As $n \rightarrow \infty$, this is the most slowly decreasing term. As predicted by Boyd [3], this amplitude is a tight bound on the asymptotic Chebyshev coefficients in the sense that the oscillating $a_{n}$ touch the curve of this endpoint contribution amplitude infinitely often as $n \rightarrow \infty$. Such a tight, bounding curve is called the envelope of the Chebyshev coefficients in [5].

The surprise is that in contrast to the asymptotic approximations of the previous two sections, which are accurate (at least in order of magnitude) even for very small $n$, the "endpoint" term in (4.2) underestimates the true coefficients (by as much as a factor of 100 ) until $n \approx 50$ and $a_{n} \sim\left(10^{-10}\right)$. The problem is that $\tanh z$ is singular at $z= \pm i \pi / 2$. (In fact, the mapping function has an infinite number of additional poles along the imaginary axis, but only the pair nearest the origin is important for $n>10$.) For moderate $n$, these pole contributions dominate the asymptotic behavior of the Chebyshev coefficients.

To dramatize this, Figure 1 also illustrates the coefficients of $g(y)$ defined by making the replacement

$$
\tanh z \rightarrow \frac{2 z}{z^{2}+\pi^{2} / 4}
$$


which has pcles at the same location at $\tanh z$. Then

$$
g(y) \equiv f(x[y])
$$

where

$$
x=\frac{2 z(y)}{[z(y)]^{2}+\pi^{2} / 4}, \quad z=\frac{L y}{\left(1-y^{2}\right)^{1 / 2}}
$$

is a function that has poles at the same location as $f(\tanh z(y))$, but is not singular at the endpoints. The short dashes, which trace the coefficients of $g(y)$, closely mimic the Chebyshev coefficients of the tanh-mapped function for $n<50$. This implies that it is the poles of the mapping-not the logarithmic singularities at the ends of the interval which motivated the mapping - which determine the magnitude of the Chebyshev coefficients for small to moderate $n$.

At the crossover point $n \approx 50$, the situation changes because the residue term proportional to $\exp (-0.48 n)$ has decayed to the magnitude of the endpoint term. The coefficients continue to fall steeply (geometrically) for $g(y)$ because its large-n coefficients have no endpoint contributions. In contrast, the coefficients of $f(\tanh z(x))$ fall more slowly because for $n>50$ they are dominated by the endpoint terms.

The crossover where the endpoint contributions come to dominate the Chebyshev coefficients occurs for smaller $n$ when the singularity is stronger. We shall return to this important issue in Section 6.

\section{APPLICATIONS. I: ASYMPTOTIC CORRECTION TO GAUSS-CHEBYSHEV QUADRATURE}

Let the $\left\{\alpha_{n}\right\}$ denote the exact Chebyshev coefficients of a function $f(x)$, and let $\left\{a_{n}\right\}$ denote the coefficients as computed by Gauss-Chebyshev quadrature with $N+1$ points in the interior of the interval. [The $\left\{a_{n}\right\}$ are the Chebyshev coefficients of the polyr mial which interpolates $f(x)$ at these points.] A well-known theorem [10] asserts

$$
\alpha_{n}=a_{n}-\sum_{j=1}^{\infty}(-1)^{j}\left[\alpha_{n+2 j(N+1)}+\alpha_{-n+2 j(N+1)}\right]
$$

If $f(x)$ is nonsingular on $x \in[-1,1]$, then the error summation is exponen- 
tially small. With boundary branch points, however, the Chebyshev coefficients will decrease as $O\left(1 / n^{k}\right)$ for some $k$. One can then show that, assuming $N \gg n$,

$$
\alpha_{n}=a_{n}-2\left(\frac{1}{2 N}\right)^{k} \sum_{j=1}^{\infty} \frac{(-1)^{j}}{j^{k}}
$$

The absolute error is $O\left(1 /[2 N]^{k}\right)$, independent of $n$. Thus, to compute the coefficients to six decimai places, one would need 1,000,000 quadrature points for $k=1$. Even then, the relative error in $a_{1000}$ would be 1 part in $10^{3}$.

However, if the asymptotic Chebyshev coefficients are known, one may substitute the asymptotic coefficients into the summation in (5.1) to evaluate it approximately. The quadrature erior is reduced by $O\left(1 / N^{2}\right)$, which is a gain of roughly a factor of 10,000 for $N$ as small as 100 .

For $k=1$-coefficients ciscreasing as $1 / n$-this "asymptotic correction" is almost essential. It was not until after this was applied that the author computed the coefficients of $\log (1-x)$ with sufficient accuracy to suspect that the asymptotic approximation was in fact exact, a conjecture subsequently confirmed by a literature search as indicated in Section 2 .

\section{APPLICATIONS. II: CROSSOVER TRUNCATION}

The mappings improve the asymptotic performance of the Chebyshev series, but for small $N$, the effect may be to reduce the rate of convergence. One must always recognize that asymptotic (as $I N \rightarrow \infty$ ) arguments only apply when $N$ is indeed large. An asymptotically "higher-order" method is superior to the Chebyshev series of the unmapped $f(x)$ only when $N$ is larger than some crossover point.

Figure 2 illustrates this theme by comparing the Chebyshev series for $f(x)=(1-x)^{2} \log (1-x)$ without a mapping with those for the same function after the applications of the sine map and tanh transformation, respectively. Asymptotically, the tanh mapping gives exponential convergence and is therefore superior to the other two series, but the graph shows that the coefficients do not become smaller thar those of the sine-mapped series until $N>70$, and the coefficients are $O\left(10^{-3}\right)$.

The other two crossover yoints show that using no coordinate transformation at all (solid curve) is superior to $t$ le sine mapping until the coefficients are as small as $O\left(10^{-4}\right)$, and to tle tanh mapping until $N=30$ and 


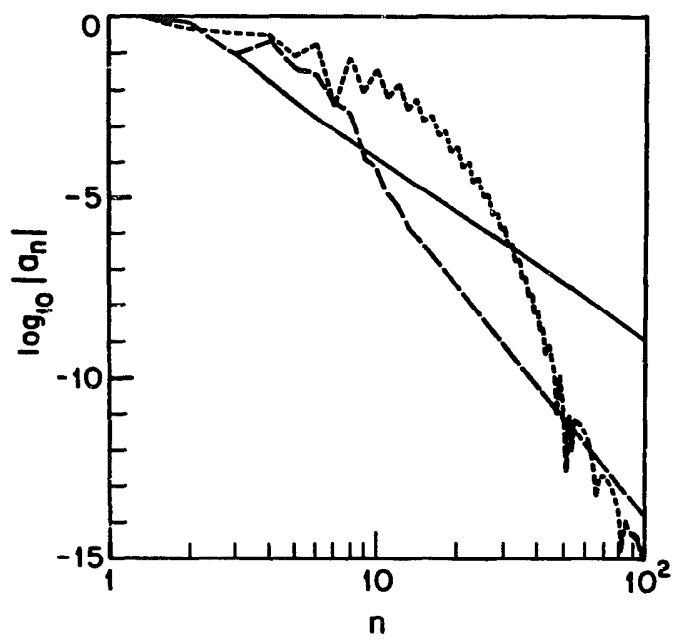

Fig. 2. Chebyshev coefficients for $f(x)=(1-x)^{2} \log (1-x)$ with no mapping (solid curve), the sine map (long dashes), and the tanh map with $L=3$ (short dashes).

$a_{n} \sim 10^{-7}$. For this weak endpoint singularity, a change of coordinate is worse than useless unless one needs very high accuracy.

For a stronger singularity as exemplified by $(1-x) \log (1-x)$, the crossover points occur for smaller $N$. In addition, one should, strictly speaking, compare errors rather than the coefficients themselves, as done in Table 2. Still, Figure 2 eloquently shows the need to apply asymptotic concepts only asymptotically.

TABLE 2

\begin{tabular}{|c|c|c|}
\hline \multicolumn{3}{|c|}{$\begin{array}{l}\text { CROSSOVER TRUNCATIONS } N_{\text {cross }} \text { WHERE ONE METHOD } \\
\text { (LEFT MEMBER OF EACH PAIR) BECOMES INFERIOR TO ANOTHER }\end{array}$} \\
\hline$N_{\text {cross }}$ & Error & Types \\
\hline \multicolumn{3}{|c|}{ (a) $f(x)=(1-x)^{2} \log (1-x)$} \\
\hline 8 & $3 E-4$ & Unmapped: sine mapping \\
\hline 28 & $3 \mathrm{E}-6$ & Unmapped: tanh mapping \\
\hline 58 & $1 \mathrm{lE}-11$ & Sine: tanh mapping \\
\hline \multicolumn{3}{|c|}{ (b) $f(x)=(1-x) \log (1-x)$} \\
\hline 4 & $3 \mathrm{E}-2$ & Unmapped: sine mapping \\
\hline 12 & $3.5 E-3$ & Unmapped : tanh mapping \\
\hline 20 & $3.5 \mathrm{E}-5$ & Sine: tanh mapping \\
\hline
\end{tabular}

${ }^{a}$ The error, which is equal for the two methods at the cross-over truncation, is the maximum pointwise error on $x \in[-1,1]\left(L_{\infty}\right.$ error). 


\section{APPLICATIONS. III: SINGULAR BASIS FUNCTIONS}

By applying Taylor-series methods as described in Bender and Orszag [1], one can show that logarithmic singularities often occur in the form of a power series multiplying the logarithm. A classic example is the Bessel function $K_{1}$, which may be written

$$
\begin{aligned}
x K_{1}(x)= & 1+\left(\log \frac{x}{2}\right)\left(\frac{x^{2}}{2}\right) \sum_{k=0}^{\infty} \frac{x^{2 k}}{4^{k} k !(k+1) !} \\
& -\frac{x^{2}}{4} \sum_{k=0}^{\infty} \frac{[\psi(k+1)+\psi(k+2)] x^{2 k}}{4^{k} k !(k+1) !}
\end{aligned}
$$

where $\psi(z)$ is the usual digamma function, the logarithmic derivative of the gamma function.

For this particular example, the coefficient of the logarithm is known to be the entire function $I_{1}(x)$. Thus, Clenshaw and Picken $[7,8]$ were able to tabulate very accurate Chebyshev series for the Bessel functions $K_{n}$ by first computing a series for the corresponding nonsingular function $I_{n}$ and then subtracting the branch point. Unfortunately, this is a special trick which is applicable to the solutions of linear, second-order ordinary differential equations and not much else.

Fortunately, the form of (7.1) suggests a numerical strategy which is broadly applicable: replacing the conventional Chebyshev basis by a mixed Chebyshev-singular basis where the singular basis functions are chosen to mimic the branch points of the desired solution, i.e.,

$$
f(x) \approx f_{N}(x)=\sum_{n=0}^{\nu} a_{n} T_{n}(x)+\sum_{k=0}^{\mu} b_{k} \phi_{k}(x)
$$

where

$$
\phi_{k}(x) \equiv(1+x)^{k} \log (1+x), \quad k=0,1, \ldots, \mu,
$$

if the singularities are at $x=-1$.

There are two disadvantages to this approach. First, it is necessary to know the precise form of the singularities. If $f(x)$ contains a term such as $(1+x)^{1 / 2} \log (1+x)$, for example, the sine mapping will be very effective, but using singular functions like (7.3) wold fail because none of the branch 
points in the basis match that of $f(x)$. (However, if we can deduce the form of the singularity, then we can appropriately generalize the singular basis functions to match.)

Second, there is no convergence theory for such mixed expansions as there is for ordinary Chebyshev series. One must make a leap of faith.

Nonetheless, numerical experiments show that this technique is very effective if the character of the endpoint singularities is known. One set of tests computed the interpolating approximation to a known, arbitrary function $f(x)$. The coefficients $\left\{a_{n}, b_{k}\right\}$ were deteimined by solving the matrix system obtained from the conditions

$$
f_{N}\left(x_{i}\right)=f\left(x_{i}\right), \quad i=0, \ldots, N
$$

where the $N+1$ interpolation points are chosen, in the absence of any theory for mixed series, to be the same as for ordinary Chebyshev collocation:

$$
x_{i}=-\cos \frac{\pi(2 i+1)}{2 N+2}, \quad i=0, \ldots, N
$$

One important practical issue: how many singular functions should be used? The theory developed in earlier sections gives a qualitative answer: only a few. The reason is that the Chebyshev series for a function like $(1+x)^{k} \log (1+x)$ converges very rapidly when $k$, the "order of the singularity," is large. Thus, quoting (2.4), one finds that for $k=6, a_{12} \approx 1.4 \times 10^{-7}$ and $a_{70} \approx 1.5 \times 10^{-17}$. In practical terms, this means that $\phi_{6}(x)$ is indistinguishable from the sum of the first 13 Chebyshev polynomials in single precision and from the sum of the first $\mathbf{7 0}$ polynomials even in double precision. Thus, using large numbers of both Chebyshev polynomials and singular functions will give a singular interpolation matrix; the basis functions, because of roundoff, will not be linearly independent.

The first test was to compute a mixed expansion for the arbitrarily chosen example

$$
f^{(1)}(x) \equiv e^{-x^{2}}+\log (1+x)\left[1+(1+x)+(1+x)^{2}\right]
$$

The exponential is included so that the function is the sum of both singular and nonsingular terms. Double-precision (17-decimal-place) calculations with 50 interpolation points and just a single singular basis function yielded $b_{0}=0.9987$, a relative error of only $0.13 \%$. The pointwise error was $\sim 10^{-8}$ everywhere except near $x=-1$, where the error is necessarily infinite unless 
$b_{0}$ is computed exactly. With 10 singular functions, $b_{0}$ was computed to a relative error of only $2.8 \times 10^{-8}, b_{1}$ to $0.005 \%$ relative error, and $b_{2}$ to $1.1 \%$.

These experiments were encouraging for two reasons. First, $f^{(1)}$ is unbounded at $x=-1$, so that conventional mapping methods will not workbut the mixed Chebyshev-singular series does. Second, ten basis functions is clearly too many, and the coefficients of $b_{3}$ to $b_{9}$ are $\sim 1$ when they should all be zero-simply numerical garbage. Nonetheless, the ill-conditioning of the basis does not prevent the calculation of an extremely accurate approximation: for all $x>0.999969$, the absolute error is no worse than $4 \times 10^{-8}$.

The second test was to apply the collocation method [6] with the same basis and grid poi 's as above to solve the boundary-value problem

$$
u_{z z}-\frac{1}{z} u_{z}-u=0, \quad u(0)=1, \quad u(\infty)=0
$$

whose exact solution is given by $u(z)=z K_{1}(z)$. The infinite interval was approximated by the large but finite interval $z \in[0, L]$, which was in turn mapped into the canonical interval $x \in[-1,1]$ via

$$
z=\frac{L}{2}(x+1) .
$$

Since the worst singularity of the exact solution is of the form $(1+x)^{2} \log (1+$ $x), \phi_{0}(x)$ and $\phi_{1}(x)$ were omitted from the basis. Although all the odd $b_{k}$ are zero for this example, $\phi_{3}(x)$ and the other odd basis functions were kept in the basis.

Table 3 shows the results. When $N=30$, where $N$ is the number of interpolation points, the maximum error in $u(x)$ decreases by a factor $\sim 100$ when $T_{29}(x)$ is replaced by $\phi_{2}(x)$, and by another two crders of magnitude when the number of singular functions is increased to 3. Using more $\phi_{k}(x)$ produces no further improvement for this $N$.

When the size of the basis is expanded to 60 , the error for all the mixed expansions was less than that of the library Bessel-function software (unfortunately single-precision) which was used for comparison. In contrast, the $L_{\infty}$ error for the pure Chebyshev series through $T_{59}(x)$ was $2 \times 10^{-5}$. At this resolution, there is an advantage to using more than three singular functions; with $M=10$, the coefficient of $(1+x)^{2} \log (1+x)$ is calculated to within 1 part in 2,000,000-a triumph.

The table also shows that the pointwise error is always much smaller then the error in the coefficients of the singular basis functions $\phi_{k}$, and this 
TABLE 3

\begin{tabular}{|c|c|c|c|c|c|}
\hline \multicolumn{6}{|c|}{ NUMERICAL SOLUTION OF THE BESSEL EQUATION ${ }^{\mathrm{a}}$} \\
\hline \multirow[b]{3}{*}{$M$} & \multicolumn{5}{|c|}{ Error } \\
\hline & \multirow{2}{*}{$\frac{L_{\infty}}{N=30}$} & \multicolumn{2}{|c|}{$b_{2}$} & \multicolumn{2}{|c|}{$b_{4}$} \\
\hline & & $N=30$ & 60 & $N=30$ & 60 \\
\hline $\mathbf{0}$ & $3.4 \mathrm{E}-4$ & - & - & - & - \\
\hline 1 & $1.2 \mathrm{E}-6$ & $0.55 \%$ & $0.029 \%$ & - & - \\
\hline 3 & $7.5 \mathrm{E}-8$ & $0.11 \%$ & $0.0012 \%$ & $20.0 \%$ & 2.4\% \\
\hline 10 & $7.6 \mathrm{E}-8$ & $0.17 \%$ & $0.000047 \%$ & $88.0 \%$ & $0.64 \%$ \\
\hline
\end{tabular}

${ }^{a} M$ is the total number of singular functions, while $N$ is the number of interpolation points. The $L_{\infty}$ error is the maximum pointwise error on $[-1,1]$. The errors in $b_{2}$ and $b_{4}$ are the relative errors in the coefficients of $(1+x)^{2} \log (1+x)$ and $(1+x)^{4} \log (1+x)$.

becomes more true as $k$ increases. In other words, the mixed series does a much better job of approximating $u(x)$ than the $b_{k}$.

The reason, as stressed above, is that the singular basis functions may be represented to some accuracy-to very high accuracy if $k$ is large-by a truncated sum of polynomials. Let

$$
\tau_{k}(\nu)=\max _{x \in[-1,1]}\left|(1+x)^{k} \log (1+x)-\sum_{n=0}^{\nu} \Phi_{n}^{(k)} T_{n}(x)\right|
$$

denote, the truncation error in the approximation of $\phi_{k}(x)$ by $\nu$ Chebyshev polynomials. Then $\tau_{k}(\nu)$ decreases rapidly with $k$ for fixed $\nu$. Let $\delta b_{k}$ denote the error in the coefficient of $\phi_{k}(x)$. Then the mixed Chebyshev-singular series can tolerate a rather large $\delta b_{k}$ if $k$ is large, because the contribution of this error to $\left|f(x)-f_{N}(x)\right|$ is weighted by $\tau_{k} \ll 1$.

In consequence, while it is clearly possible to use the mixed expansion to compute the coefficients of at least the lowest two logarithmic singularities for the Bessel function $K_{1}$, the computed coefficients of large-k singular basis functions are merely random numbers. In these and other experiments not reported here, we found it was almost never useful to include more than five singular functions in the basis. We employed as many as $\mathbf{1 0}$ here only to show that the ill-conditioning problem does not seriously degrade the pointwise error.

In spite of the lack of a rigorous theory, Table 3 proves that the mixed expansion can be very successful. Replacing one or two Chebyshev polynomi- 
als (out of 60) does not significantly reduce the resolution at the center of the interval.

In contrast, mapping methods have two disadvantages. First, the change of coordinate lacks the ability to isolate the branch points and compute the strengths of at least the lowest two or three singularities. Second, mapping extracts the price that the extra resolution near the endpoints implies much poorer resolution on the interior of the computational domain.

\section{SUMMARY}

Endpoint singularities seriously degrade the convergence of a Chebyshev series. In this article, we have made this poor convergence precise for logarithmic singularities by deriving asymptotic approximations to the Chebyshev coefficients as $n \rightarrow \infty$.

A change of coordinate that gives higher resolution near the endpoints can greatly improve convergence. We have quantified this improvement for two particular mappings. One is algebraic (the sine transformation), and the other is exponential in the sense that the new coordinate varies exponentially with the old in the neighborhood of the endpoints.

The exponential (tanh) mapping, similar to one suggested by Stenger [14], is always superior in the asymptotic limit $n \rightarrow \infty$ in the sense that the Chebyshev coefficients of the mapped function decrease exponeniially fast with $n$, the degree of the coefficient. However, for finite $n$, the sine mapping seems to be preferable. Although the coefficients decrease only as an algebraic function of $n$-an inverse power of $n$-the power of $n$ is so large that the sine mapping gives smaller error than the tanh mapping unless the truncation $N$ is very large. The weakness of the tanh change of coordinate is that resolution is reduced too drastically near the center of the interval.

In contrast, the sine map is both effective and simple. As long as the singularity is bounded $-\log (1-x)$ multiplied by $(1-x)^{k}$ for $k>0$ rather than a "naked" logarithm-it is possible to obtain very good accuracy with Chebyshev series and the sinusoidal change of coordinate.

An alternative strategy is to replace a small number (we suggest no more than five) of the Chebyshev polynomials by singular basis functions whose form is chosen to match the most singular branch points in the solution of the differential equation. (It is not necessary to know the coefficients of the singular terms, only the form.) This tactic demands a much more detailed knowledge of the character of the endpoint singularities than is true of the mapping methods. Furthermore, there is no rigorous convergence theory for such mixed Chebyshev-singular series. Nonetheless, the numerical experiments reported in Section 7 show that singular basis functions may be 
extremely effective. The strength of this method is that the singularities are neutralized without significantly reducing resolution near the center of the interval.

Clearly, there are good tools for coping with endpoint singularities. It is our belief that both the sine mapping and sir.gular basis functions will find uses in real-world problems. Some two-dimensional applications in fluid mechanics are described in Lee, Schultz, and Boyd [12].

This work was supported by the National Science Foundation under Grant OCE8509923 and DMS8716766.

\section{REFERENCES}

I C. M. Bender and S. A. Crszag, Advanced Mathematical Methods for Scientists and Engineers, McGraw-Hill, New York, 1978, pp. 281-282.

2 J. P. Boyd, Polynomial series versus sinc expansions for functions with corner or endpoint singularities, J. Comput. Phys. 64:266-269 (1986).

3 J. P. Boyd, The optimization of convergence for Chebyshev polynomial methods in an unbounded domain. I. Comput. Phys. 45:43-79 (1982).

4 J. P. Boyd, Spectral methods using rational basis functions on an infinite interval, J. Comput. Phys. 69:112-143 (1987).

5 J. P. Boyd, An analytical and numerical study of the two-dimensional Bratu equation, J. Sci. Comput. 1:183-206 (1986).

6 J. P. Boyd, Spectral Methods, Springer, New York, 1989.

7 C. W. Clenshaw, Chebyshev series for mathematical functions, Math. Tables 5:1-36 (1962).

8 C. W. Clenshaw and S. M. Pickens, Chebyshev series for Bessel functions of fractional order, Math. Tables 8:1-60 (1966).

9 D. Elliott, The evaluation and estimation of the coefficients in the Chebyshev series expansion of a function, Math. Comp. 18:274-284 (1964).

10 L. Fox and I. Parker, Chebyshev Polynomials in Numerical Analysis, Oxford U.P., London, 1968.

11 I. S. Gradshteyn and I. M. Ryzhik, Table of Integrals, Series, and Products, 4th ed., Academic, New York, 1965, p. 38.

12 N.-Y. Lee, and W. W. Schultz, and J. P. Boyd, Chebyshev pseudospectral method for viscous flows with corner singularity, J. Sci. Comput., submitted for publication.

13 I. R. Lund and B. V. Riley, A sinc-collocation method for the computation of the eigenvalues of the radial Schrödinger equation, IMA J. Numer. Anal. 4:83-98 (1984),

14 F. Stenger, Numerical methods based on Whittaker cardinal, or sinc functions, SIAM Rev. 23:165-224 (1981). 